\title{
Importance of the correct assessment of bone fractures in the clinical management of metastatic castration-resistant prostate cancer treated with radium-223: A case report
}

\author{
ELISA LODI RIZZINI $^{1,2}$, PIETRO GHEDINI ${ }^{1,2}$, RAFFAELE CARDANO $^{2,3}$, CHIARA BELLAROSA $^{2,3}$, \\ ALESSIO GIUSEPPE MORGANTI $^{2,3}$, STEFANO FANTI ${ }^{1,2}$ and FABIO MONARI $^{3}$ \\ ${ }^{1}$ Nuclear Medicine Unit, S. Orsola-Malpighi Hospital; ${ }^{2}$ DIMES University of Bologna; \\ ${ }^{3}$ Radiation Oncology Center, S. Orsola-Malpighi Hospital, I-40138 Bologna, Italy
}

Received July 9, 2018; Accepted March 27, 2019

DOI: $10.3892 / \mathrm{mco} .2019 .1852$

\begin{abstract}
Patients who undergo radium-223 treatment for metastatic castration-resistant prostate cancer (mCRPC) generally have a long history of androgen deprivation therapy and/or steroid therapy, which leads to bone loss and causes osteoporosis. Notably, Osteoporosis in combination with metastatic bone disease increases the risk of bone fracture. An 84-year-old man with multi-metastatic bone CRPC underwent six administrations of intravenous radium-223, which induced a good biochemical and clinical response. However, two months following the treatment, the patient reported acute pain localized to the lumbar spine mimicking bone progression disease and presented with stable prostate-specific antigen levels. A prostate-specific membrane antigen-positron emission tomography scan showed no tracer uptake in that site, whereas a magnetic resonance imaging scan and subsequent vertebral biopsy confirmed the absence of cancer progression and showed the presence of vertebral crushing of L4-L5, which was probably due to an osteoporotic process. The patient had never received bisphosphonate therapy and refused it during $\alpha$-emitting therapy with radium-223. The osteoporotic process, in association with metastatic bone
\end{abstract}

Correspondence to: Dr Elisa Lodi Rizzini, Nuclear Medicine Unit, S. Orsola-Malpighi Hospital, Via Massarenti 9, I-40138 Bologna, Italy

E-mail: elisalodirizzini@libero.it

Abbreviations: mCRPC, metastatic castration-resistant prostate cancer; BMD, bone mineral density; ALP, alkaline phosphatase; 99mTc-BS, technetium-99m bone scintigraphy; PSMA-PET, prostate-specific membrane antigen-positron emission tomography; MRI, magnetic resonance imaging; PSA, prostate-specific antigen; SSE, symptomatic skeletal event; NRS, Numeric Rating Scale

Key words: $\alpha$-emitting therapy, radium-223, metastatic castrationresistant prostate cancer, osteoporosis, bone fractures, bisphosphonates therapy disease, more easily leads to bone fractures that have an important impact on performance status, quality of life and prognosis quoad vitam in patients with advanced prostate cancer. Use of bisphosphonates or anti-RANKL antibody appears to be effective in improving bone mineral density. Notably, patients with multi-metastatic bone disease who undergo radium-223 therapy should be treated in conjunction with anti-osteoporotic therapy (bisphosphonates or anti-RANKL antibody) and adequate calcium and vitamin D supplementation. Early recognition and differentiation of osteoporotic processes when determining the progression of cancer-associated bone disease is crucial in evaluating the response to radium-223 therapy and, consequently, for further therapeutic decision making.

\section{Introduction}

Patients with metastatic castration-resistant prostate cancer (mCRPC) treated with radium-223 have osteoblastic and/or osteolytic symptomatic/mildly symptomatic bone disease; bone metastases correlate with increased risk of symptomatic skeletal adverse events (1). In addition, such patients generally have a long history of androgen deprivation therapy and/or steroid therapy [co-administration of which is recommended, for example, in association with the hormonal agent abiraterone acetate (2) for treatment of prostate cancer] that leads to bone weakening and causes osteoporosis. The association of these two factors with the presence of metastatic bone lesions elevates the risk of bone fractures and decreases the time to symptomatic skeletal events (SSE), defined as the first use of external-beam radiation therapy to relieve skeletal symptoms, new symptomatic pathologic vertebral or non-vertebral bone fractures, spinal cord compression, or tumor-related orthopedic surgical intervention (3). This process negatively affects not only prognosis quoad vitam but also results in a significant deterioration in the quality of life (4-6). For this reason, it is essential to understand which patients are at higher risk of osteoporosis and to provide, where possible, adequate therapy with a bisphosphonate or anti-RANKL antibody and calcium/vitamin D supplementation to prevent or treat the osteoporotic process (7-9). 


\section{Case report}

M.G.C., an 84-year-old man, had received a biopsy diagnosis of prostate adenocarcinoma, with a Gleason Score of $7(4+3)$ in 2005 at the age of 72 and was treated with an exclusive external radiation therapy to the prostate gland (total dose $=76 \mathrm{~Gy}$ in 38 fractions) and monthly hormonal therapy with leuprorelin $\left(\right.$ Enantone $\left.^{\circledR}\right) 3.75 \mathrm{mg}$. In his remote clinical history, there was a right eye enucleation for a non-specific uveitis process in 1948, a coronary angioplasty for a cardiologic pathology in 1998 , hypercholesterolemia and hepatitis B virus-related chronic liver disease. In 2011 he underwent brachytherapy on the prostate gland (total dose $=23 \mathrm{~Gy}$ ) for a local relapse documented by biopsy and external radiation therapy to pelvic lymph nodes (total dose $=58.8 \mathrm{~Gy}$ in 28 fractions), and bicalutamide was added to his androgen deprivation therapy. At the end of 2016, elevation of prostate-specific antigen (PSA) level and a technetium- $99 \mathrm{~m}$ bone scintigraphy ( $99 \mathrm{mTc}-\mathrm{BS})$ documented progression of bone disease with the appearance of multiple bone metastasis to the skull, the spine, to the ribs bilaterally and to the pelvis (Fig. 1A and B). There was no evidence of visceral metastases and/or lymphadenopathy greater than $3 \mathrm{~cm}$ at restaging computed tomography.

The patient, who also reported pain in the spine, particularly the cervical spine [Numeric Rating Scale (NRS)=3], was then scheduled for alpha-emitting therapy and from 21 December 2016 to 11 May 2017 he underwent six administrations of intravenous radium-223, with good clinical tolerance to therapy. In January 2017, after the first radium-223 cycle, he underwent external radiation therapy to the cervical spine, with good pain response (NRS from 3 to 0/1). Because of the presence of multi-metastatic disease and his long history of androgen deprivation therapy, he was evaluated by a maxillo-facial specialist, who recommended that zoledronic acid therapy should be started. However, this was refused by the patient, who also declined supplementation with calcium and vitamin D. During the six administrations of radium-223, the pains related to the spine gradually decreased (from $2 / 3$ to $0 / 1$ on a NRS), without the need for any pharmacological analgesic therapy. There was also a $>30 \%$ decrease of alkaline phosphatase (ALP) values, showing an ALP response to radium-223 therapy (3).

Furthermore, a $99 \mathrm{mTc}-\mathrm{BS}$ performed one month after the end of radium-223 treatment showed a slight diffuse decrease of tracer uptakes on metastatic bones. Only a faint tracer uptake on L4-L5 appeared, which was attributed to an inflammatory/osteodegenerative process (Fig. 1C and D). Nevertheless, only two months after the end of treatment, the patient reported the onset of acute lumbar sciatica $(\mathrm{NRS}=7 / 8)$ partially responsive to pharmacological analgesic therapy. For this reason, on 14 July 2017, a magnetic resonance imaging (MRI) scan showed a vertebral crushing on the L4-L5 level with spongiotic bone edema (Fig. 2). A subsequent prostate-specific membrane antigen-positron emission tomography (PSMA-PET) scan showed no pathologic tracer uptake on the same vertebral level (Fig. 3). In August 2017, the patient, after orthopedic consultation, underwent vertebroplasty on L4-L5 with a vertebral biopsy that confirmed the absence of neoplastic bone infiltration. After surgical intervention the patient had a moderate decrease of the pain (NRS from $7 / 8$ to 5 ).
One year after the end of radium-223 treatment, the patient had stable biochemical disease and there was slight uptake decrease of the metastatic bone lesions at PET-PSMA scan. No other oncology therapies have been administered.

\section{Discussion}

Patients with advanced prostate cancer with skeletal localizations have an increased risk of developing bone fractures. This risk is further increased if associated with osteoporosis induced by prolonged androgen deprivation therapy and/or steroid therapy (5).

The Alpharadin in Symptomatic Prostate Cancer Patients (ALSYMPCA) study was a phase 3, randomized, double-blind, multinational study that compared the efficacy and safety of radium-223 with that of placebo in patients with CRPC and bone metastases. The ALSYMPCA trial showed that the time to the first SSE was significantly prolonged with radium-223 (median, 15.6 months vs. 9.8 months; $\mathrm{HR}=0.66$; $\mathrm{P}<0.001$ ) (3) and that concomitant bisphosphonate therapy strongly predicted decreased risk of SSE, including bone fractures (1). This effect may be as a result of an additive effect of the two agents in reducing SSE via independent different mechanisms, but may also be explained by the bisphosphonate-related inhibition of osteoclasts leading to an accumulation of inorganic bone matrix that could increase radium-223 uptake $(1,10)$.

All currently available bisphosphonates are effective in improving bone mineral density (BMD) compared to placebo in prostate cancer patients (11) although zoledronic acid has been shown to be associated with a greater improvement in BMD at all bone sites assessed (12). Furthermore, beginning zoledronic acid therapy one month prior to the first administration of radium-223 may increase the likelihood of pain palliation and prevent pain flare (13). Parker et al reported an increase in survival induced by radium-223 therapy in $\mathrm{mCRPC}$, with a median survival duration of 14.0 months compared with 11.2 months with placebo (HR: 0.70; $\mathrm{P}=0.002)$ (3). The RANK-ligand-inhibitor, denosumab, has also been shown to improve clinical outcome when administered concomitantly with radium-223; in the international expanded access program for radium-223, patients receiving combination treatment denosumab plus radium-223 show longer survival (median not available vs. 13 months with radium-223 alone), on the contrary there wasn't a survival benefit with the bisphosphonates combination (14). Currently, there is emerging evidence from post hoc analyses of data from pivotal phase 3 studies that bisphosphonate therapy in combination with radium-223 may also increase survival, as well as contributing to the prevention of SSE (15). For this reason, it is important to consider beginning bone-targeted therapies early in patients with multi-metastatic bone prostate cancer on treatment with alpha-emitting therapy. Because there is limited information about the prevention of osteoporosis (16-18), supplementation with calcium and vitamin $\mathrm{D}$ in these patients is also recommended, as in non-oncology patients with osteoporosis $(4,19)$. Furthermore, early recognition and differentiation of osteoporotic processes from bone disease progression becomes crucial in accurately assessing response to radium-223 therapy and consequently for further therapeutic decision making. In 

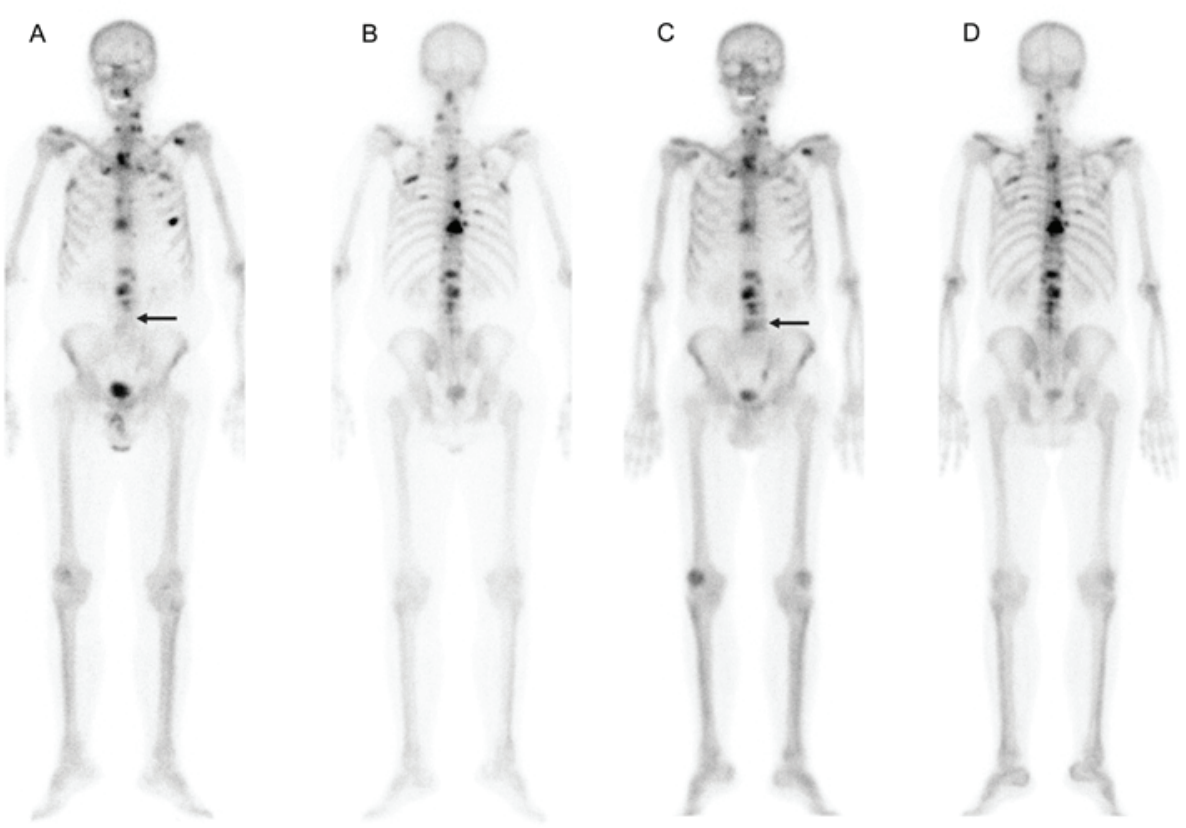

Figure 1. (A and B) Pre-radium-223 treatment (19 Dec, 2016) and (C and D) post-radium-223 treatment (23 Jun, 2017) 99mTc-BS. In scans (A) and (C), the arrows indicated the appearance of a faint tracer uptake on L4-L5, which was attributed to an inflammatory/osteodegenerative process.

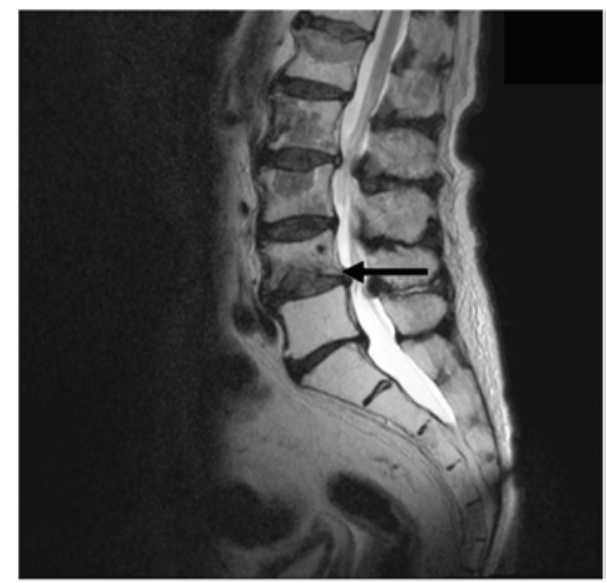

Figure 2. Magnetic resonance imaging scan post-radium-223 treatment showed vertebral crushing on L4 (arrow).

our case, the patient refused to start zoledronic acid therapy despite the recommendation of a maxillo-facial specialist, and two months after the end of radium-223 therapy he showed a lumbar vertebral crushing that mimicked bone progression disease at scintigraphy bone scan and through the clinical manifestation of acute lumbar pain. However, PSMA-PET and MRI scanning helped us to differentiate neoplastic from osteoporotic disease (20-24). This, in association with a stable PSA value and a decreased ALP value, which was shown to have predictive value in the ALSYMPCA trial $(25,26)$, directed us to request an orthopedic opinion which subsequently led to identifying the correct therapeutic strategy to follow the radium-223 treatment.

In conclusion, patients with CRPC and bone metastases who are enrolled for radium-223 therapy frequently have concomitant osteoporotic disease due to prolonged hormone therapy and/or steroid therapy. The osteoporotic process can increase

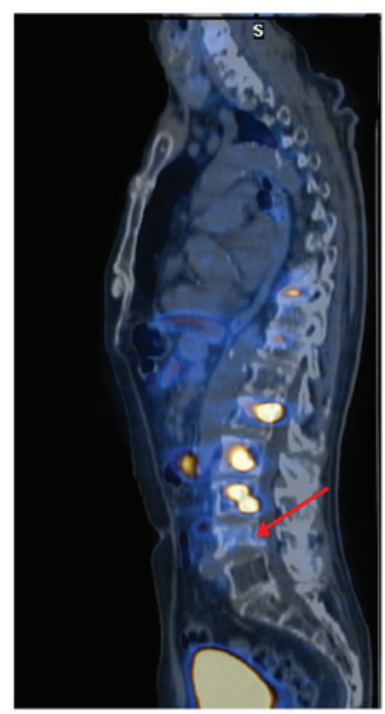

Figure 3. Prostate-specific membrane antigen-positron emission tomography scan post-radium-223 treatment. There was no pathologic tracer uptake on L4-L5 (red arrow).

the risk of or accelerate the development of symptomatic skeletal events which lead to considerable morbidity and deterioration in the quality of life. Furthermore, complications of osteoporosis, mimicking SSE due to bone progression disease, can interfere with the response evaluation to alpha-emitting therapy and affect future therapeutic decisions in patients with metastatic prostate cancer. For this reason, to limit the osteoporotic process, it is important to start with bone-targeted therapies such as bisphosphonates or denosumab, along with adequate prophylactic calcium/vitamin D supplementation, as soon as possible during radium-223 treatment. This is particularly important for patients with multi-metastatic disease and at low risk of mandibular osteonecrosis. 


\section{Acknowledgements}

Not applicable.

\section{Funding}

No funding was received.

\section{Availability of data and materials}

The datasets used and/or analyzed during the current study are available from the corresponding author on reasonable request.

\section{Authors' contributions}

ELR (first author) analyzed clinical and imaging data of the patient and wrote main part of the article, PG contributed to analysis, in particular imaging evaluation. RC, CB, AGM, FM and SF were involved in the clinical management of the patients and contribute with imaging and other clinical data.

\section{Ethics approval and consent to participate}

Written informed consent was obtained from the patient.

\section{Patient consent for publication}

The patient provided written informed consent for publication of the present study.

\section{Competing interests}

The authors declare that they have no competing interests.

\section{References}

1. Sartor O, Coleman R, Nilsson S, Heinrich D, Helle SI, O'Sullivan JM, Fosså SD, Chodacki A, Wiechno P, Logue J, et al: Effect of radium-223 dichloride on symptomatic skeletal events in patients with castration-resistant prostate cancer and bone metastases: Results from a phase 3, double-blind, randomised trial. Lancet Oncol 15: 738-746, 2014.

2. European Medicines Agency: Zytiga (abiraterone acetate) Summary of product characteristics. https://www.ema.europa.eu/ en/medicines/human/EPAR/zytiga. Accessed September 23, 2011

3. Parker C, Nilsson S, Heinrich D, Helle SI, O'Sullivan JM, Fosså SD, Chodacki A, Wiechno P, Logue J, Seke M, et al: Alpha emitter radium-223 and survival in metastatic prostate cancer. N Engl J Med 369: 213-223, 2013.

4. Rosino-Sánchez A: Preventive management of bone disease in advanced prostate cancer. Arch Esp Urol 71: 258-266, 2018 (In Spanish).

5. Smith MR: Osteoporosis and other adverse body composition changes during androgen deprivation therapy for prostate cancer. Cancer Metastasis Rev 21: 159-166, 2002.

6. Smith MR: Diagnosis and management of treatment-related osteoporosis in men with prostate carcinoma. Cancer 97 789-795, 2003.

7. Tanvetyanon T: Physician practices of bone density testing and drug prescribing to prevent or treat osteoporosis during androgen deprivation therapy. Cancer 103: 237-241, 2005.

8. Diamond TH, Higano CS, Smith MR, Guise TA and Singer FR: Osteoporosis in men with prostate carcinoma receiving androgen-deprivation therapy: Recommendations for diagnosis and therapies. Cancer 100: 892-899, 2004.
9. Coleman RE and McCloskey EV: Bisphosphonates in oncology. Bone 49: 71-76, 2011.

10. Cha TL, Wu TT, Vogelzang NJ, Huang CY, Huang SP, Lin CC, Ou YC, Pang ST, Shen DH, Wu WJ and Chang WY: Optimal usage of radium-223 in metastatic castration-resistant prostate cancer. J Formos Med Assoc 116: 825-836, 2017.

11. Macherey S, Monsef I, Jahn F, Jordan K, Yuen KK, Heidenreich A and Skoetz N: Bisphosphonates for advanced prostate cancer. Cochrane Database Syst Rev 12: CD006250, 2017.

12. Poon Y, Pechlivanoglou P, Alibhai SMH, Naimark D, Hoch JS, Papadimitropoulos E, Hogan ME and Krahn M: Systematic review and network meta-analysis on the relative efficacy of osteoporotic medications: Men with prostate cancer on continuous androgen-deprivation therapy to reduce risk of fragility fractures. BJU Int 121: 17-28, 2018.

13. Yap KKL, Wong W, Ji L, Groshen SG, Quinn DI, Pinski JK, Bryce $\mathrm{AH}$ and Dorff TB: Impact of timing of administration of bone supportive therapy on pain palliation from radium-223. J Clin Oncol 35 (Suppl 15): S5023, 2017.

14. Saad F, Carles J, Gillessen S, Heidenreich A, Heinrich D, Gratt J, Lévy J, Miller K, Nilsson S, Petrenciuc O, et al: Radium-223 and concomitant therapies in patients with metastatic castration-resistant prostate cancer: An international, early access, open-label, single-arm phase 3b trial. Lancet Oncol 17: 1306-1316, 2016.

15. Saad F, Sternberg CN, Mulders PFA, Niepel D and Tombal BF: The role of bisphosphonates or denosumab in light of the availability of new therapies for prostate cancer. Cancer Treat Rev 68: 25-37, 2018

16. Heidenreich A, Bastian PJ, Bellmunt J, Bolla M, Joniau S, van der Kwast T, Mason M, Matveev V, Wiegel T, Zattoni F, et al: EAU guidelines on prostate cancer. Part II: Treatment of advanced, relapsing, and castration-resistant prostate cancer. Eur Urol 65: 467-479, 2014.

17. Saylor PJ: Bone targeted therapies for the prevention of skeletal morbidity in men with prostate cancer. Asian J Androl 16: 341-347, 2014.

18. Morgans AK and Smith MR: Bone-targeted agents: Preventing skeletal complications in prostate cancer. Uro Clin North Am 39: 533-546, 2012.

19. Bilezikian JP: Osteoporosis in men. J Clin Endocrinol Metab 84: 3431-3434, 1999.

20. Udovicich C, Perera M, Hofman MS, Siva S, Del Rio A, Murphy DG and Lawrentschuk N: ${ }^{68} \mathrm{Ga}$-prostate-specific membrane antigen-positron emission tomography/computed tomography in advanced prostate cancer: Current state and future trends. Prostate Int 5: 125-129, 2017.

21. von Eyben FE, Picchio M, von Eyben R, Rhee H and Bauman G: ${ }^{68} \mathrm{Ga}$-labeled prostate-specific membrane antigen ligand positron emission tomography/computed tomography for prostate cancer: A systematic review and meta-analysis. Eur Urol Focus 4: 686-693, 2018

22. Osman OM, Fahmy YR, El-Oraby AM, El-Basmy AA and Amin YE: Role of diffusion WIs and T2* GRE pulse sequences in dubious vertebral marrow pathological lesions. J Egypt Natl Canc Inst 19: 254-262, 2007.

23. Cicala D, Briganti F, Casale L, Rossi C, Cagini L, Cesarano E, Brunese L and Giganti M: Atraumatic vertebral compression fractures: Differential diagnosis between benign osteoporotic and malignant fractures by MRI. Musculoskelet Surg 97 (Suppl 2): S169-S179, 2013

24. Brodowicz T, Hadji P, Niepel D and Diel I: Early identification and intervention matters: A comprehensive review of current evidence and recommendations for the monitoring of bone health in patients with cancer. Cancer Treat Rev 61: 23-34, 2017.

25. Sartor O, Coleman RE, Nilsson S, Heinrich D, Helle SI, O'Sullivan JM, Vogelzang NJ, Bruland $\varnothing$, Kobina S, Wilhelm S, et al: An exploratory analysis of alkaline phosphatase, lactate dehydrogenase, and prostate-specific antigen dynamics in the phase 3 ALSYMPCA trial with radium-223. Ann Oncol 28: 1090-1097, 2017.

26. Nguyen NC, Shah M, Appleman LJ, Parikh R and Mountz JM: Radium-223 therapy for patients with metastatic castrate-resistant prostate cancer: An update on literature with case presentation. Int J Mol Imaging 2016: 2568031, 2016. 